\title{
Acupuncture for the Treatment of Knee Osteoarthritis: An Overview of Systematic Reviews
}

\author{
Jixin Chen (iD) $1,2, *$ \\ Aifeng Liu ${ }^{1,2, *}$ \\ Qinxin Zhou ${ }^{1,2}$ \\ Weijie Yu ${ }^{l, 2}$ \\ Tianci Guo ${ }^{1,2}$ \\ Yizhen Jia ${ }^{1,2}$ \\ Kun Yang ${ }^{1,2}$ \\ Puyu Niu ${ }^{1,2}$ \\ Huichuan Feng ${ }^{1,2}$ \\ 'Department of Orthopaedic Surgery, \\ First Teaching Hospital of Tianjin \\ University of Traditional Chinese \\ Medicine, Tianjin, 30038I, People's \\ Republic of China; ${ }^{2}$ National Clinical \\ Research Center for Chinese Medicine \\ Acupuncture and Moxibustion, Tianjin, \\ 30038I, People's Republic of China
}

*These authors contributed equally to this work
Correspondence: Aifeng Liu Department of Orthopaedic Surgery, First Teaching Hospital of Tianjin University of Traditional Chinese

Medicine, No. 88 Changling Road, Xiqing

District, Tianjin, 30038I, People's

Republic of China

Tel +86-I380309|533

Email draifeng@।63.com
Background: Acupuncture has been widely used in the clinical management of osteoarthritis of the knee (KOA). Many systematic reviews (SRs) and meta-analyses (MAs) have reported its effectiveness in relieving pain. This overview aimed to summarize SRs and MAs on the effectiveness and safety of acupuncture for KOA and evaluate their methodological and evidence quality of the included SRs and MAs.

Methods: We conducted a comprehensive literature search for SRs and MAs in four Chinese and four international databases from their inception until August 2021. Two researchers independently searched the reviews, extracted the data, and cross-checked the data. The Assessing the Methodological Quality of Systematic Reviews 2 (AMSTAR 2) tool was used to evaluate the methodological quality of the included SRs and MAs. The Grades of Recommendations, Assessment, Development, and Evaluation (GRADE) system was used to assess the quality of evidence for the outcomes of the included SRs and MAs.

Results: A total of 14 SRs and MAs were included. The evaluation results of the AMSTAR 2 tool showed that the methodological quality of all the 14 SRs and MAs was critically low. The principal causes are the lack of a pre-registration proposal and a list of excluded studies and justify the exclusions, the report on the sources of funding, and the reasons for the study designs for inclusion. The results of the GRADE evaluation showed 25 of 46 outcomes were very low-level evidence. Seventeen were of low level, four were of moderate level and none were of high level. Most outcomes were downgraded in quality of evidence mainly because of publication bias and imprecision.

Conclusion: The existing evidence suggests that acupuncture seems to be an effective and safe therapy for KOA. However, the deficiencies in the methodological quality and quality of evidence of the included SRs/MAs have limited the reliability of the conclusions. Therefore, further rigorous and comprehensive studies are warranted to verify the effectiveness and safety of acupuncture in KOA.

Keywords: acupuncture, knee osteoarthritis, overview, AMSTAR 2, GRADE, systematic review

\section{Introduction}

Knee osteoarthritis (KOA) is a degenerative disorder with the main clinical manifestations of pain, joint swelling, functional limitation, and even disability. ${ }^{1}$ The incidence of KOA shows an increasing trend in recent years, and the disease has

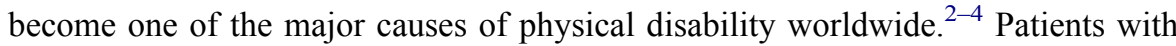
pain and limited mobility are prone to negative emotions such as anxiety, which greatly affects their quality of life. To date, there is no radical cure for KOA, and 
the treatment mainly aims to relieve pain and restore joint function, with a commitment to reducing the disability rate. In the latest guidelines of the management of KOA released by the American College of Rheumatology, exercise, self-management, strength training, weight loss, use of canes and knee pads, topical NSAIDs, and intraarticular injections of glucocorticoids are strongly recommended for all patients with KOA. ${ }^{5}$ Analgesics alone for pain control, although effective, can cause not only liver and kidney damage in the long term, but also lead to overuse of the joints and increase joint damage and degeneration. ${ }^{6}$ There is still reservation about the longterm safety and efficacy of NSAIDs and opioids, which will cause significant gastrointestinal reactions, cardiovascular reactions and hepatic and renal toxicities. ${ }^{7-9}$ According to the guidelines, oral glucocorticoids are not considered, and chondroprotective agents such as glucosamine and chondroitin sulphate are not effective on KOA patients. ${ }^{10,11}$ The long-term efficacy of oral antioxidants or vitamin D remains controversial, while intra-articular hormone injections can also accelerate articular cartilage degeneration, although they can relieve pain and improve joint function. ${ }^{12}$ Besides, intra-articular injections such as sodium vitreous acid are ineffective on patients with severe KOA. ${ }^{13,14}$ Total knee arthroplasty (TKA) is mainly indicated for the treatment of severe KOA, with a high incidence of complications. ${ }^{15}$ Neither biological nor tissue-engineered restorative treatments are applied in clinic at present. ${ }^{11,12,16}$

Acupuncture is an important component of traditional Chinese medicine (TCM) and an effective alternative therapy for relieving pains. ${ }^{17,18}$ It is associated with fewer adverse effects than conventional approaches. ${ }^{19,20}$ As many people with KOA have difficulties in exercise and weight loss, acupuncture is conditionally recommended for KOA. Several studies have suggested the potential benefits of acupuncture to generally improve symptoms such as joint pain, swelling and stiffness in KOA. ${ }^{21-23}$ The theoretical biochemical basis of acupuncture includes the release of various endogenous substances to relieve pain. ${ }^{24-26}$ In recent years, numerous RCTs, SRs and MAs on the acupuncture treatment of KOA have been published to compare the effectiveness and safety of acupuncture treatment with control group. However, the results of SRs and MAs are conflicting, and the conclusions are comprised by the sample size and included study quality. ${ }^{27,28}$ However, no consensus has been reached and their quality has not been evaluated yet, which is an indispensable step before treatment recommendations can confidently be made. A systemic review is a new approach designed to synthesize the results from multiple SRs and MAs. ${ }^{29}$ To overcome the limitations of an individual SR and to provide comprehensive evidence, a systemic review on SRs and MAs is required.

Several recent $\mathrm{SRs}^{27,28,30}$ have evaluated acupuncture interventions since the publication of the first systemic review in $2019 .{ }^{31}$ Hence, to fully appraise the available data, we updated the prior analyses and included studies published during the last 3 years. To draw the most reliable conclusion possible, the PubMed database search was also conducted, and only SRs/MAs including randomized controlled trials (RCTs) were considered. This review aimed to comprehensively evaluate the methodological quality of these SRs and MAs using the Assessing the Methodological Quality of Systematic Reviews $2^{32}$ (AMSTAR 2) tool, access the quality of the latest evidence of important outcomes from the included MAs using the Grades of Recommendations, Assessment, Development, and Evaluation ${ }^{33}$ (GRADE) system, and summarize the conclusions of these SRs and MAs to further clarify the safety and effectiveness.

\section{Materials and Methods}

The protocol of this overview was registered on the International Prospective Register of Systematic Reviews (PROSPERO; http://www.crd.york.ac.uk/PROSPERO/; registration number, CRD42021277210). This overview of SRs/MAs was performed in accordance with guidelines introduced by the Cochrane Collaboration Search Strategy and Preferred Reporting Items for Systematic Reviews and Meta-Analyses (PRISMA) statement.

\section{Search Strategy}

Electronic literature searches were conducted by 2 independent researchers in four international electronic databases (PubMed, EMBASE, Cochrane Library, and Web of Science) and four Chinese electronic databases (China National Knowledge Infrastructure Database, Wanfang database, Chinese Biomedical Literature Database and Chongqing VIP) from inception to July 31, 2021. No restrictions were made on publication time and language. The search terms were as follows: ("osteoarthritis of the knee" OR "knee osteoarthritis" OR "koa" OR "gonarthritis" OR "knee pain" AND ("acupuncture" OR "acupuncture therapy" OR "manual acupuncture" OR "electroacupuncture" OR "auricular acupuncture" OR "warm acupuncture" OR 
“acupoints" OR "filiform needle" OR "fire needle") AND ("systematic assessment" OR "systematic review" OR "meta-analysis"). In addition, we manually searched for any pertinent articles to ensure that the data collection was comprehensive. The detailed search strategy in PubMed is shown in Supplemental Table A.

\section{Inclusion Criteria}

We included SRs/MAs that matched the following criteria:

- Study Design: SRs/MAs included RCTs of acupuncture for patients with KOA and performed metaanalysis.

- Study Participants: Patients were diagnosed with KOA clinically or radiographically without restriction on sex, age, and race. ${ }^{11,12}$

- Study Intervention: Treatment group intervention included various acupuncture therapy (acupuncture, electroacupuncture, auricular acupuncture, warm acupuncture, etc.) and acupuncture combined with other therapies.

- Study Comparison: Control group intervention included comfort therapy (placebo, sham acupuncture or blank control) and other therapies (medication therapy or nondrug therapy, etc.).

- Study Outcome Measures: At least one of the prespecified outcomes was reported: effective rate, cure rate, Western Ontario and McMaster Universities Arthritis Index scores (WOMAC), visual analog scale (VAS), adverse effects and other indicators. ${ }^{34,35}$

\section{Exclusion Criteria}

- Non-major intervention of acupuncture in the treatment group or acupuncture as an intervention in the control group.

- Inclusion of non-randomized controlled trial literature within SRs/MAs.

- Duplicate published literature.

- Non-SRs/MAs, comments, conference abstracts, and studies on which the data could not be extracted.

\section{Literature Screening and Data Extraction}

According to the search strategy, two researchers imported the retrieved literature titles into Endnote X8 software. After removing duplicates, two researchers independently read the titles and abstracts of the articles to exclude studies that clearly did not meet the inclusion criteria, and then read the full text of the studies that might meet the inclusion criteria for screening. Data extraction included first author, publication year, country, the number of included studies, sample size, interventions, control measures, outcomes, quality assessment methods and conclusions. Data extraction was respectively performed by two researchers, and conflicts were resolved through discussion and consultation with a third author. We attempted to contact authors to obtain further information that we may have missed, as necessary.

\section{Assessment of Systematic Reviews}

The quality of included studies was evaluated by two authors independently according to the corresponding standards. Disagreements were resolved by consensus or discussions with the third author.

\section{Assessment of Methodological Quality}

Two reviewers independently assessed the quality of included SRs/MAs by AMSTAR 2 tool, which consists of 16 items, among which seven (items 2, 4, 7, 9, 11, 13, and 15) are critical. Each item is evaluated by 3 criteria: "Yes", "Partially Yes", and "No". According to the evaluation results and the criticality of the entries, four quality levels are evaluated: high, moderate, low and critically low. $\leq 1$ non-critical entry defect is rated as high, $>1$ noncritical entry defect is rated as medium, 1 critical entry defect with or without non-critical entry defect is rated as low, $>1$ critical entry defect with or without non-critical entry defect is rated as critically low.

\section{Grading of the Quality of Evidence}

Two reviewers independently applied the GRADE (Grades of Recommendation, Assessment, Development and Evaluation) system to evaluate the quality of evidence for outcomes of included SRs/MAs. Evidence is graded as high based on RCTs and might be downgraded due to the following 5 criteria: risk of bias, inconsistencies, indirectness, inaccuracy, and publication bias. The quality of evidence was classified as high, moderate, low, and critically low.

\section{Assessment of Bias}

Two researchers independently employed the ROBIS tool to evaluate the risk of bias in the included SRs/MAs. ${ }^{36}$ The five critical evaluation categories were: "study inclusion criteria", "study retrieval and screening", "data extraction and quality assessment", "data integration and presentation of results", and overall risk of bias of SRs/MAs. Each domain was evaluated as "high risk," "low risk," or "unclear risk." 


\section{Results}

\section{Literature Search and Selection}

A total of 481 related literatures were collected from 8 electronic databases. Two hundred and eight were excluded because of duplication, and 259 were excluded on the basis of the title or abstract. After reading the full text, 10 of the remaining 24 literatures were excluded. After being reviewed by two reviewers independently, 14 SRs/MAs on acupuncture for KOA were included (Figure 1). ${ }^{37-50}$ The excluded literatures and reasons for exclusion are shown in Supplemental Table B.

\section{Characteristics of the SRs/MAs}

The characteristics of the 14 SRs/MAs can be found in Table $1 .{ }^{37-50}$ All SRs/MAs included only RCTs and were published between 2009 and 2021, including four studies $^{38,41,47,48}$ published in English and ten studies $^{37,39,40,42-46,49,50}$ published in Chinese. Ten studies $^{38,41-49}$ were employed by using the Cochrane risk of bias criteria to evaluate the quality of research, 4 studies $^{37,39,40,50}$ were employed by using the Jadad scale. The number of studies included in each SRs/MAs ranged from 6 to 21 , and the total participants ranged from 500 to 3552 . The treatment group intervention measures were mostly acupuncture therapy (AT), warm acupuncture (WA), fire needle acupuncture (FA), electro-acupuncture (EA) or acupuncture combined with other therapies. The control group intervention measures were mainly medication therapy, sham acupuncture, sodium hyaluronate intra-articular injection, no treatment or placebo.

\section{Methodological Quality}

The results of AMSTAR 2 tool are shown in Table 2. The qualities of 14 SRs/MAs were considered critically low, because they had more than one critical flaw (items 2, 4, 7, $9,11,13$, and 15) with multiple non-critical flaws. The key factors affecting the methodological quality of the SRs/MAs were item 2 (none of studies reported their prior study protocols), item 3 (all studies did not explain the type of study included), item 7 (none of studies provided a list of reasons

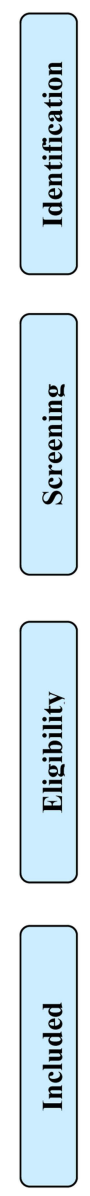

Records identified through Chinese database $(\mathrm{n}=306)$ CNKI $(n=130)$, WF $(n=69)$,

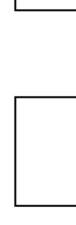

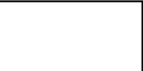

Records identified through English database $(\mathrm{n}=185)$

Web of science $(n=27)$, Embase $(n=84)$, PubMed $(n=73)$, Cochrane library $(n=1)$

Records after duplicates removed $(\mathrm{n}=208)$

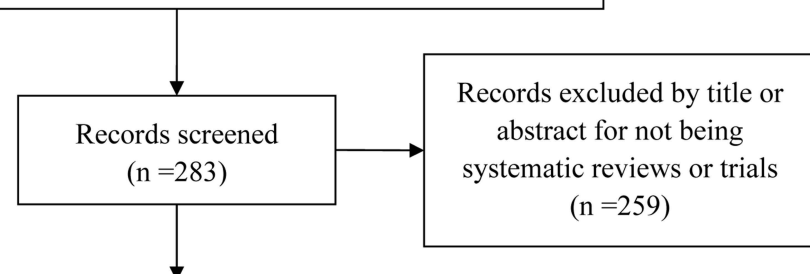

Full-text articles assessed for eligibility $(\mathrm{n}=24)$

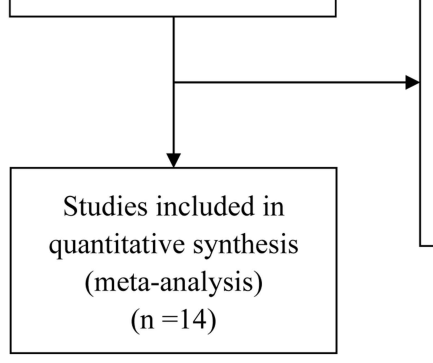

Full-text articles excluded, with reasons $(\mathrm{n}=10)$

1 Non-RCT not only RCT

5 The control group does not meet the requirements 4 The treatment group does not meet the requirements

Figure I Flowchart of the selection process of included SRs and MAs. 


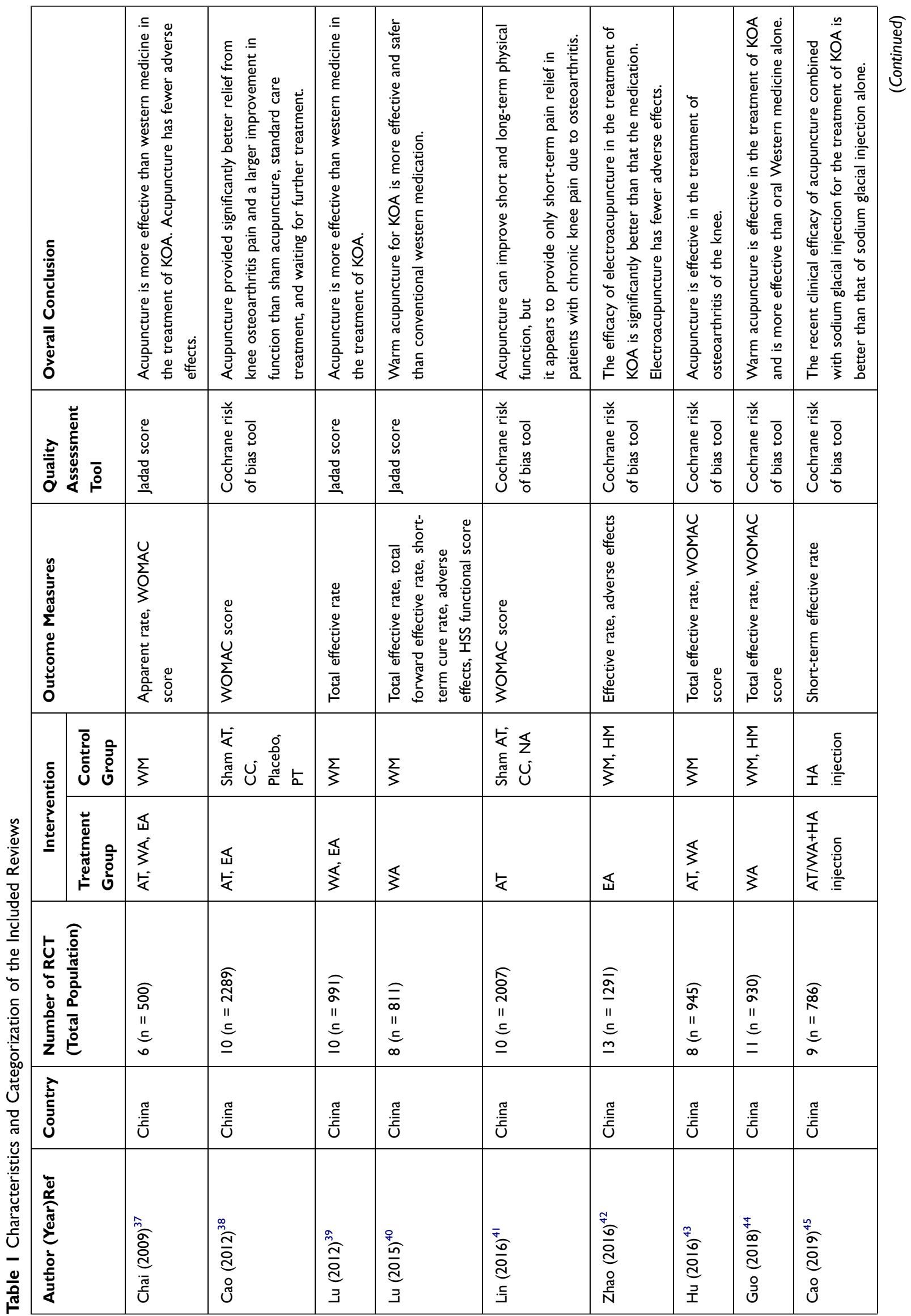




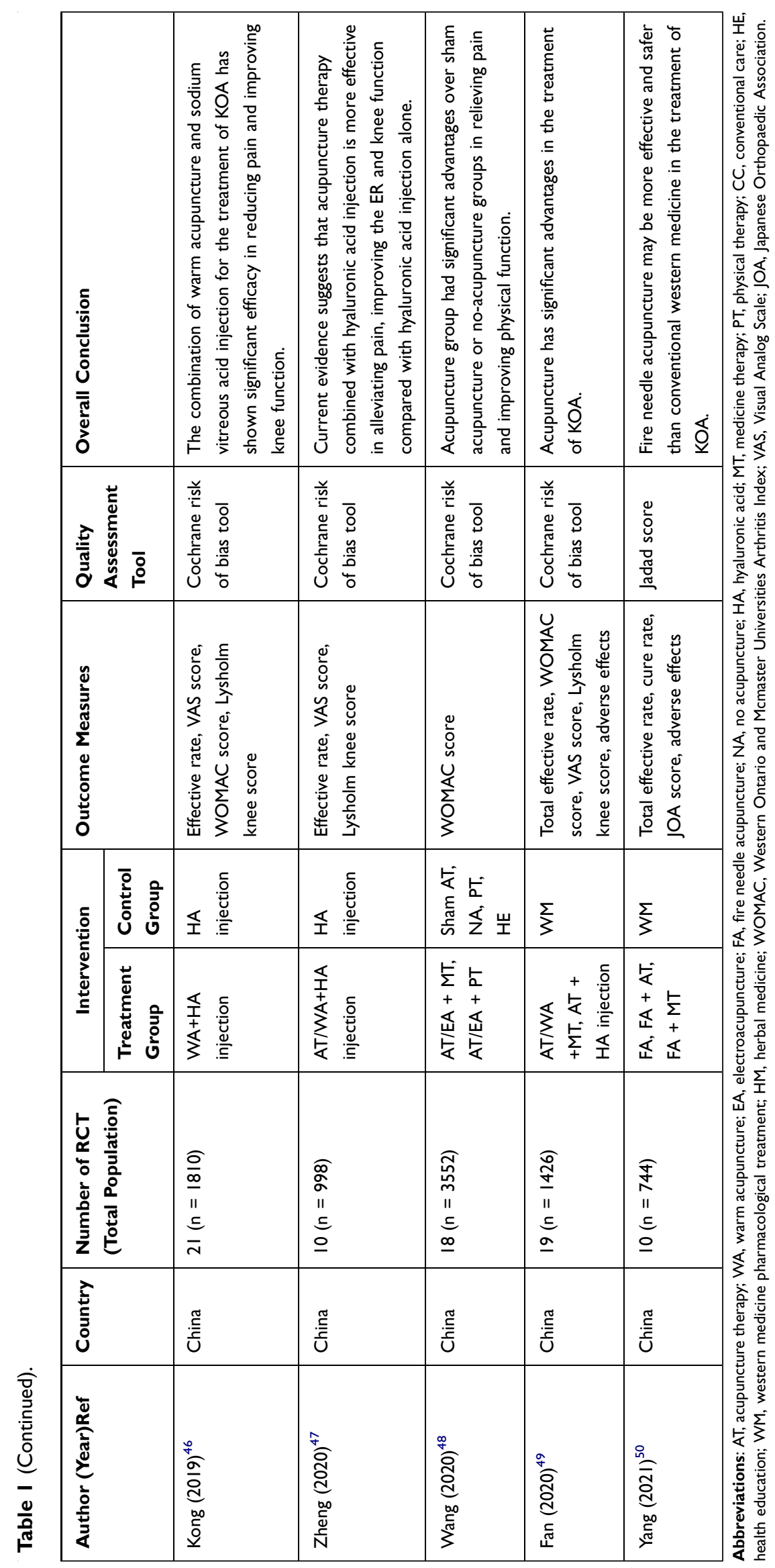




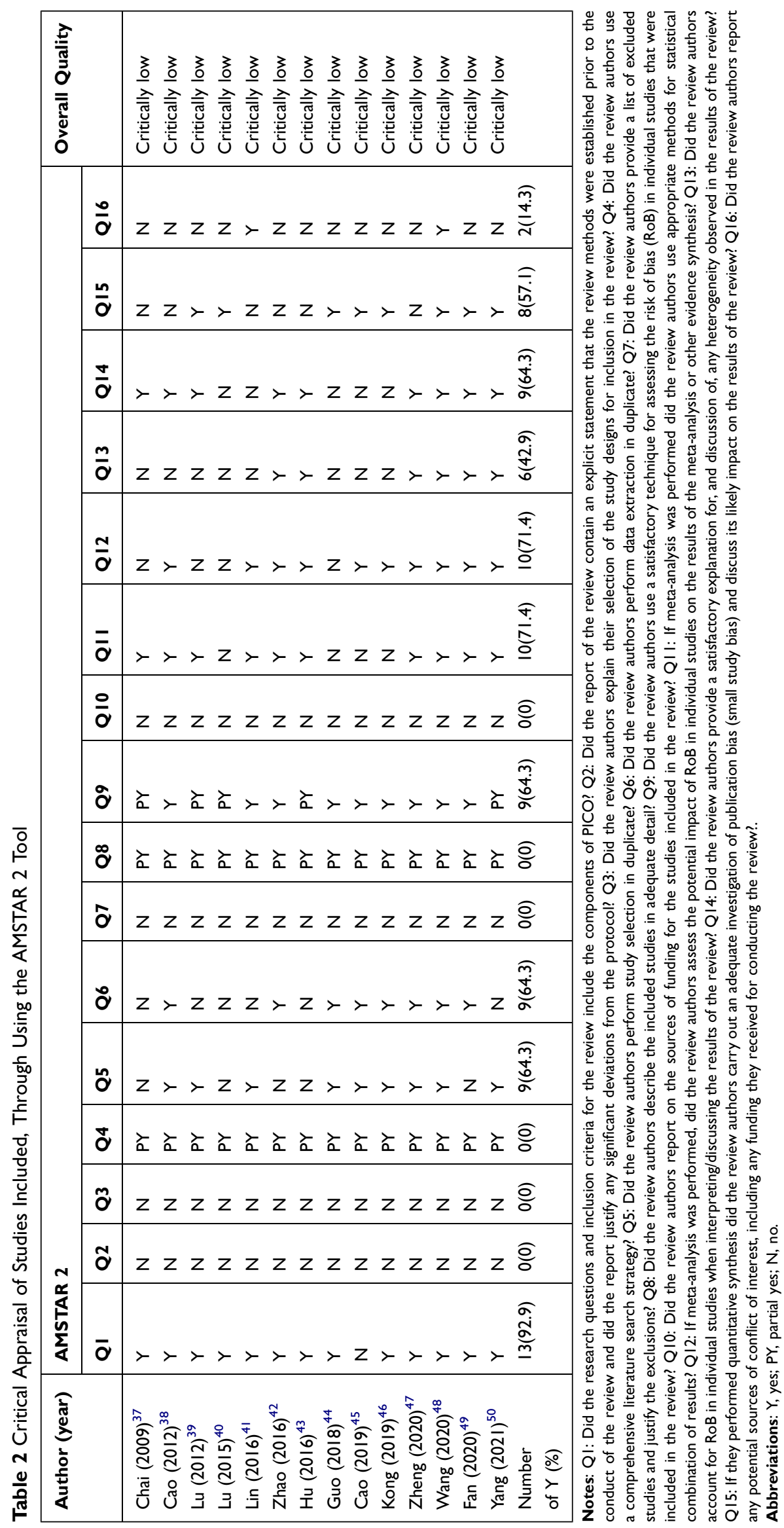


Table 3 GRADE Quality Grading of I4 SRs and MAs of Acupuncture for KOA

\begin{tabular}{|c|c|c|c|c|c|c|c|}
\hline Author(Year) & Outcomes (n) & Limitations & Inconsistency & Indirectness & Imprecision & $\begin{array}{c}\text { Publication } \\
\text { Bias }\end{array}$ & $\begin{array}{l}\text { Quality } \\
\text { of } \\
\text { Evidence }\end{array}$ \\
\hline Chai $(2009)^{37}$ & $\begin{array}{l}\text { Apparent rate (4) } \\
\text { WOMAC score (2) } \\
\text { WOMAC pain scores (2) } \\
\text { WOMAC stiffness score (2) } \\
\text { WOMAC daily activity } \\
\text { score (2) }\end{array}$ & $\begin{array}{l}-1^{(1)} \\
-I^{1} \\
-I^{(1)} \\
-I^{(1)} \\
-I^{(1)}\end{array}$ & $\begin{array}{l}0 \\
0 \\
0 \\
0 \\
0\end{array}$ & $\begin{array}{l}0 \\
0 \\
0 \\
0 \\
0\end{array}$ & $\begin{array}{l}0 \\
0 \\
0 \\
0 \\
0\end{array}$ & $\begin{array}{l}-1^{(5)} \\
-1^{(5)} \\
-1^{(5)} \\
-1^{(5)} \\
-1^{(5)}\end{array}$ & $\begin{array}{l}\text { Low } \\
\text { Low } \\
\text { Low } \\
\text { Low } \\
\text { Low }\end{array}$ \\
\hline Cao $(2012)^{38}$ & $\begin{array}{l}\text { AT versus SA for pain and } \\
\text { function, short-term (10) } \\
\text { AT versus SA for pain and } \\
\text { function, long-term (4) } \\
\text { AT versus UC for pain and } \\
\text { function, short-term (6) } \\
\text { AT versus UC for pain and } \\
\text { function, long-term (4) } \\
\text { AT versus WS for pain and } \\
\text { function, short-term (5) }\end{array}$ & $\begin{array}{l}-I^{(1)} \\
-I^{(1)} \\
-I^{(1)} \\
-I^{(1)} \\
-I^{(1)}\end{array}$ & $\begin{array}{l}-1^{(2)} \\
0 \\
-1^{(2)} \\
0 \\
-1^{(2)}\end{array}$ & $\begin{array}{l}0 \\
0 \\
0 \\
0 \\
0\end{array}$ & $\begin{array}{c}0 \\
-I^{(3)} \\
0 \\
-I^{(3)} \\
0\end{array}$ & $\begin{array}{l}-1^{(5)} \\
-1^{(5)} \\
-1^{(5)} \\
-1^{(5)} \\
-1^{(5)}\end{array}$ & $\begin{array}{l}\text { Very Low } \\
\text { Very Low } \\
\text { Very Low } \\
\text { Very Low } \\
\text { Very Low }\end{array}$ \\
\hline Lu $(2012)^{39}$ & Total efficacy rate (10) & $-I^{(1)}$ & 0 & 0 & 0 & $-1^{(4)}$ & Low \\
\hline $\operatorname{Lu}(2015)^{40}$ & $\begin{array}{l}\text { Short-term total efficacy } \\
\text { rate }(8) \\
\text { Long-term total efficacy } \\
\text { rate }(2) \\
\text { Short-term cure rate (4) } \\
\text { Adverse effect }(2)\end{array}$ & $\begin{array}{l}-1^{(1)} \\
-I^{(1)} \\
-I^{(1)} \\
-I^{1}\end{array}$ & $\begin{array}{c}-1^{(2)} \\
0 \\
0 \\
0\end{array}$ & $\begin{array}{l}0 \\
0 \\
0 \\
0\end{array}$ & $\begin{array}{l}0 \\
0 \\
0 \\
0\end{array}$ & $\begin{array}{l}0 \\
0 \\
0 \\
0\end{array}$ & $\begin{array}{l}\text { Low } \\
\text { Moderate } \\
\text { Moderate } \\
\text { Moderate }\end{array}$ \\
\hline $\operatorname{Lin}(2016)^{41}$ & $\begin{array}{l}\text { Short-term WOMAC } \\
\text { physical function scores } \\
\text { (I0) } \\
\text { Long-term WOMAC } \\
\text { physical function scores (5) } \\
\text { Short-term WOMAC pain } \\
\text { scores (II) } \\
\text { Long-term WOMAC pain } \\
\text { scores (5) }\end{array}$ & $\begin{array}{l}-\mathbf{I}^{(1)} \\
-\mathbf{I}^{(1)} \\
-\mathbf{I}^{(1)} \\
-\mathbf{I}^{(1)}\end{array}$ & $\begin{array}{l}-2^{(2)} \\
-2^{(2)} \\
-2^{(2)} \\
-2^{(2)}\end{array}$ & $\begin{array}{l}0 \\
0 \\
0\end{array}$ & $\begin{array}{c}0 \\
-1^{(3)} \\
-1^{(3)}\end{array}$ & $\begin{array}{l}0 \\
0 \\
0\end{array}$ & $\begin{array}{l}\text { Very Low } \\
\text { Very Low } \\
\text { Very Low } \\
\text { Very Low }\end{array}$ \\
\hline Zhao $(2016)^{42}$ & $\begin{array}{l}\text { Efficacy rate }(13) \\
\text { Adverse effect }(1)\end{array}$ & $\begin{array}{l}-1^{(1)} \\
-I^{(1)}\end{array}$ & $\begin{array}{l}0 \\
0\end{array}$ & $\begin{array}{l}0 \\
0\end{array}$ & $\begin{array}{l}0 \\
0\end{array}$ & $\begin{array}{l}-1^{(5)} \\
-1^{(5)}\end{array}$ & $\begin{array}{l}\text { Low } \\
\text { Low }\end{array}$ \\
\hline $\mathrm{Hu}(2016)^{43}$ & $\begin{array}{l}\text { Efficacy rate (8) } \\
\text { WOMAC score (2) }\end{array}$ & $\begin{array}{l}-I^{1} \\
-I^{(1)}\end{array}$ & $\begin{array}{c}0 \\
-1^{(2)}\end{array}$ & $\begin{array}{l}0 \\
0\end{array}$ & $\begin{array}{l}0 \\
0\end{array}$ & $\begin{array}{l}-1^{(5)} \\
-1^{(5)}\end{array}$ & $\begin{array}{c}\text { Low } \\
\text { Very Low }\end{array}$ \\
\hline Guo $(2018)^{44}$ & $\begin{array}{l}\text { Total efficacy rate (II) } \\
\text { WOMAC score ( } 2)\end{array}$ & $\begin{array}{l}-1^{(1)} \\
-I^{(1)}\end{array}$ & $\begin{array}{c}0 \\
-2^{(2)}\end{array}$ & $\begin{array}{l}0 \\
0\end{array}$ & $\begin{array}{c}0 \\
-I^{(3)}\end{array}$ & $\begin{array}{l}-1^{(4)} \\
-1^{(4)}\end{array}$ & $\begin{array}{c}\text { Low } \\
\text { Very Low }\end{array}$ \\
\hline Cao $(2019)^{45}$ & Short-term efficacy rate (9) & $-I^{(1)}$ & 0 & 0 & 0 & 0 & Moderate \\
\hline Kong $(2019)^{46}$ & $\begin{array}{l}\text { Efficacy rate (I6) } \\
\text { VAS score (I4) } \\
\text { Lysholm knee score (II) } \\
\text { WOMAC score (5) }\end{array}$ & $\begin{array}{l}-I^{1} \\
-I^{1} \\
-I^{1} \\
-I^{1}\end{array}$ & $\begin{array}{c}0 \\
-2^{(2)} \\
-2^{(2)} \\
-2^{(2)}\end{array}$ & $\begin{array}{l}0 \\
0 \\
0 \\
0\end{array}$ & $\begin{array}{l}0 \\
0 \\
0 \\
0\end{array}$ & $\begin{array}{l}-I^{(4)} \\
-I^{(5)} \\
-I^{(5)} \\
-I^{(5)}\end{array}$ & $\begin{array}{l}\text { Low } \\
\text { Very Low } \\
\text { Very Low } \\
\text { Very Low }\end{array}$ \\
\hline
\end{tabular}

(Continued) 
Table 3 (Continued).

\begin{tabular}{|c|c|c|c|c|c|c|c|}
\hline Author(Year) & Outcomes (n) & Limitations & Inconsistency & Indirectness & Imprecision & $\begin{array}{c}\text { Publication } \\
\text { Bias }\end{array}$ & $\begin{array}{l}\text { Quality } \\
\text { of } \\
\text { Evidence }\end{array}$ \\
\hline Zheng $(2020)^{47}$ & $\begin{array}{l}\text { Efficacy rate ( } 8) \\
\text { VAS score (7) } \\
\text { Lysholm knee score }(7)\end{array}$ & $\begin{array}{l}-I^{(1)} \\
-I^{(1)} \\
-I^{1}\end{array}$ & $\begin{array}{c}0 \\
-2^{(2)} \\
-2^{(2)}\end{array}$ & $\begin{array}{l}0 \\
0 \\
0\end{array}$ & $\begin{array}{l}0 \\
0 \\
0\end{array}$ & $\begin{array}{l}-1^{(5)} \\
-1^{(5)} \\
-1^{(5)}\end{array}$ & $\begin{array}{l}\text { Low } \\
\text { Very Low } \\
\text { Very Low }\end{array}$ \\
\hline Wang $(2020)^{48}$ & $\begin{array}{l}\text { AT versus NA for pain } \\
\text { reduction (II) } \\
\text { AT versus NA for physical } \\
\text { function improvement (I0) } \\
\text { AT versus SA for pain } \\
\text { reduction (I2) } \\
\text { AT versus SA for physical } \\
\text { function improvement (I2) }\end{array}$ & $\begin{array}{l}-1^{(1)} \\
-1^{(1)} \\
-1^{(1)} \\
-1^{(1)}\end{array}$ & $\begin{array}{l}-2^{(2)} \\
-2^{(2)} \\
-2^{(2)} \\
-2^{(2)}\end{array}$ & $\begin{array}{l}0 \\
0 \\
0 \\
0\end{array}$ & $\begin{array}{l}-1^{(3)} \\
-1^{(3)} \\
-1^{(3)} \\
-1^{(3)}\end{array}$ & $\begin{array}{l}0 \\
0 \\
0 \\
0\end{array}$ & $\begin{array}{l}\text { Very Low } \\
\text { Very Low } \\
\text { Very Low } \\
\text { Very Low }\end{array}$ \\
\hline Fan $(2020)^{49}$ & $\begin{array}{l}\text { Total efficacy rate (19) } \\
\text { Lysholm knee score (4) } \\
\text { VAS score (II) } \\
\text { WOMAC score (16) } \\
\text { Adverse effect (8) }\end{array}$ & $\begin{array}{l}-I^{(1)} \\
-I^{(1)} \\
-I^{(1)} \\
-I^{(1)} \\
-I^{(1)}\end{array}$ & $\begin{array}{c}0 \\
-2^{(2)} \\
-2^{(2)} \\
-2^{(2)} \\
0\end{array}$ & $\begin{array}{l}0 \\
0 \\
0 \\
0 \\
0\end{array}$ & $\begin{array}{c}0 \\
0 \\
0 \\
0 \\
-1^{(3)}\end{array}$ & $\begin{array}{l}-1^{(4)} \\
-1^{(4)} \\
-1^{(4)} \\
-1^{(4)} \\
-1^{(4)}\end{array}$ & $\begin{array}{l}\text { Very Low } \\
\text { Very Low } \\
\text { Very Low } \\
\text { Very Low } \\
\text { Very Low }\end{array}$ \\
\hline Yang $(202 \mathrm{I})^{50}$ & $\begin{array}{l}\text { total efficacy rate }(10) \\
\text { Short-term cure rate }(10) \\
\text { Adverse effect }(1) \\
\text { JOA score }(3)\end{array}$ & $\begin{array}{l}-I^{(1)} \\
-I^{(1)} \\
-I^{1} \\
-I^{(1)}\end{array}$ & $\begin{array}{l}0 \\
0 \\
0 \\
0\end{array}$ & $\begin{array}{l}0 \\
0 \\
0 \\
0\end{array}$ & $\begin{array}{l}-1^{(3)} \\
-1^{(3)} \\
-I^{(1)} \\
-1^{(3)}\end{array}$ & $\begin{array}{l}0 \\
0 \\
0 \\
0\end{array}$ & $\begin{array}{l}\text { Low } \\
\text { Low } \\
\text { Low } \\
\text { Low }\end{array}$ \\
\hline
\end{tabular}

Notes: ${ }^{(1)}$ The design of the experiment with a large bias in random, distributive hiding or blind; ${ }^{(2)}$ The confidence interval overlaps less, the heterogeneity test $P$ is very small, and the $l^{2}$ is larger; ${ }^{3}$ Confidence interval is not narrow enough; ${ }^{(4)}$ Funnel graph asymmetry; ${ }^{(5)}$ Fewer studies are included and there may be greater publication bias. Abbreviations: AT, acupuncture therapy; NA, no acupuncture; SA, sham acupuncture; UC, usual care; WS, waiting list; WOMAC, Western Ontario and Mcmaster Universities Arthritis Index; VAS, Visual Analog Scale; JOA, Japanese Orthopaedic Association.

for excluding studies), item 10 (none of studies reported on the sources of funding for the studies included), item 16 (none of studies report any potential sources of conflict of interest).

\section{Grading of the Quality of Evidence}

The 14 SRs/MAs included 46 outcomes of the effectiveness and safety of acupuncture for KOA. The results showed that a total of 25 outcome indicators were of very low quality of evidence, 17 were of low quality, 4 were of moderate quality, and none was of high quality, as detailed in Table 3. Limitations (100\%) and publication bias (63.0\%) were the main reasons for downgrading.

\section{The Effectiveness and Safety of Acupuncture for KOA}

According to the moderate-quality evidence, $1 \mathrm{SR}^{40}$ suggested that acupuncture combined with hyaluronic acid injection was better than hyaluronic acid injection alone with short-term effective rate (risk ratio $[R R]=1.16,95 \%$ confidence interval [CI] [1.10, 1.22], $\mathrm{P}<0.00001) .1 \mathrm{SR}^{45}$ reported that warm acupuncture treatment was more effective compared to western medicine measured with Long-term total efficacy rate $(\mathrm{RR}=1.16,95 \%[\mathrm{CI}][1.04,1.29], \mathrm{P}=0.008)$ and Short-term cure rate $(\mathrm{RR}=2.35,95 \%[\mathrm{CI}][1.59,3.45], \mathrm{P}<0.0001)$. This review also reported less adverse effect than western medicine $(\mathrm{RR}=0.20,95 \%[\mathrm{CI}][0.05,0.75], \mathrm{P}=0.02)$. The available evidence suggests that acupuncture appears to be an effective and safe therapy for KOA.

\section{Risk of Bias}

The ROBIS tool was used to assess the risk of bias of included SRs/MAs. Domain 1 assessed concerns regarding specification of study eligibility criteria, 12 SRs/MAs $(85.7 \%)^{37-42,44,46-50}$ were rated as low risk of bias in the domain 1, which regarding the study eligibility criteria. 4 SRs/MAs $(28.6 \%)^{39,44,46,47}$ were rated as low risk of bias in the domain 2, which 
regarding identification and selection of studies. 7 SRs/ MAs $(50 \%)^{38,44-49}$ were rated as low risk of bias and one review was unclear risk of bias in domain 3, which regarding data collection and study appraisal. 4 SRs/ MAs $(28.6 \%)^{39,48-50}$ were rated as low risk of bias in domain 4 , which regarding the synthesis and findings. The final phase considered the overall risk of bias of SRs/MAs, and 7 SRs/MAs $(50 \%)^{40,41,45-48,50}$ were rated as low-risk of bias. The assessment of the risk of bias of each review is shown in Table 4 and Figure 2.

\section{Discussion}

\section{Summary of Main Findings}

$\mathrm{SRs} / \mathrm{MAs}$ are the integrative analyses of the original research evidence, which are at a higher level in the evidence hierarchy. The main conclusion in this work was that acupuncture treatment was advantageous in relieving pain and improving knee joint function. With regard to safety, there was no serious adverse effect associated with acupuncture. Numerous RCTs and systematic evaluations have also confirmed the effectiveness and safety of acupuncture in the treatment of KOA. ${ }^{19,22,23,27,30,31,51}$ However, the quality of methodological evaluations and outcome indicators of 14 SRs/MAs was low and very low.

\section{Assessment of the Quality of the Included SRs and MAs}

As shown by the methodological quality evaluation of the AMSTAR 2 scale in the current work, the methodological quality of all the included SRs/MAs regarding the application of acupuncture in KOA was extremely low. None of the enrolled SRs/MAs offered a pre-protocol, or described manual searches and grey literature searches. Besides, all the systematic reviews failed to describe in detail the essential characteristics of the included studies, which made it impossible to compare the studies at baseline. Further, none of the enrolled systematic reviews provided a list of excluded literature. All our enrolled SRs/MAs did not offer a list of the excluded literature or information on the excluded literature was not available. In our enrolled SRs/MAs, reasonable tools were utilized to evaluate the risk of bias among the studies, among which, 4 employed the Jadad scale $37,39,40,50$ and 10 applied the Cochrane Risk of Bias Assessment Tool. ${ }^{38,41-49}$ None of our enrolled SRs/MAs described funding support, and only one mentioned the relevant conflicts of interest. In this regard, the SRs/MAs researchers should pay more attention to the above-mentioned issues and improve the methodological quality, so as to provide a high-quality basis for guideline development and clinical decision-making.

In this work, the GRADE evidence rating system was employed to grade the quality of individual outcome

Table 4 Tabular Presentation of Risk of Bias of Included SRs/MAs

\begin{tabular}{|c|c|c|c|c|c|}
\hline \multirow[t]{2}{*}{ Review } & \multicolumn{4}{|c|}{ Phase 2} & \multirow{2}{*}{\begin{tabular}{l}
\multicolumn{1}{c}{ Phase 3} \\
Risk of Bias in \\
the Review
\end{tabular}} \\
\hline & $\begin{array}{l}\text { I. Study } \\
\text { Eligibility } \\
\text { Criteria }\end{array}$ & $\begin{array}{l}\text { 2. Identification and } \\
\text { Selection of Studies }\end{array}$ & $\begin{array}{l}\text { 3. Data Collection and } \\
\text { Study Appraisal }\end{array}$ & $\begin{array}{l}\text { 4.Synthesis } \\
\text { and Findings }\end{array}$ & \\
\hline Chai $(2009)^{37}$ & ;) & : : & :) & : : & : \\
\hline Cao $(2012)^{38}$ & (:) & (:) & (:) & (2) & (:) \\
\hline $\operatorname{Lu}(2012)^{39}$ & (:) & (:) & (:) & (:) & (:) \\
\hline Lu $(2015)^{40}$ & (:) & (:) & (2) & (2) & (:) \\
\hline $\operatorname{Lin}(2016)^{41}$ & (:) & (:) & (:) & (:) & ;) \\
\hline Zhao $(2016)^{42}$ & (:) & (:) & $?$ & (2) & (:) \\
\hline $\mathrm{Hu}(2016)^{43}$ & : $:$ & (:) & (:) & (:) & :) \\
\hline Guo $(2018)^{44}$ & (:) & (:) & (:) & :) & (:) \\
\hline Cao $(2019)^{45}$ & (2) & (2) & (-) & :) & (:) \\
\hline Kong $(2019)^{46}$ & (:) & (:) & (:) & (2) & (:) \\
\hline Zheng $(2020)^{47}$ & (:) & (:) & (:) & (2) & (:) \\
\hline Wang $(2020)^{48}$ & (:) & (2) & (:) & ;) & ;) \\
\hline Fan $(2020)^{49}$ & (:) & (:) & (:) & (:) & : : \\
\hline Yang $(202 \mathrm{I})^{50}$ & ;) & :) & :) & (:) & ;) \\
\hline
\end{tabular}

Abbreviations: $:$, low risk of bias; $:$, high risk of bias; ?, unclear. 


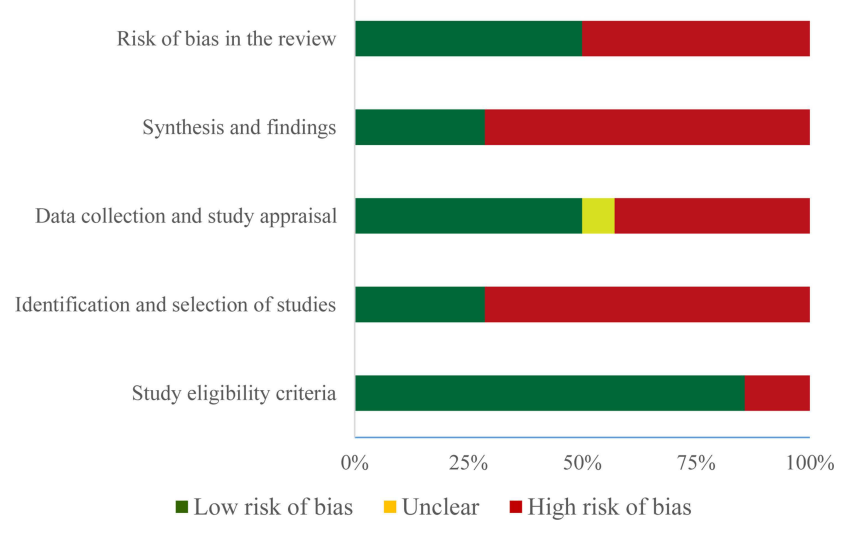

Figure 2 Graphical presentation of risk of bias of included SRs and MAs.

indicators. According to the obtained results, 25 outcome indicators were of extremely low quality of evidence, 17 of low quality, 4 of medium quality, while 0 of high quality, and there was little variability in the quality of individual indicators. The majority of indicators were of very low quality of evidence, indicating that there were variations in the study outcomes. Typically, imprecision and publication bias in the studies were the major causes of most of the downgrades, followed by inconsistency and limitations. Of them, imprecision was mainly ascribed to the wide confidence intervals (CIs) or the insufficient sample size of included studies and the poor overlap of CIs. Besides, publication bias was mainly reflected by the asymmetric funnel plots, insufficiently narrow CIs, and inclusion of studies that did not meet the sample size estimation requirements for clinical trials or studies with potential publication bias. On the other hand, inconsistency was reflected in the poor overlap of CIs between studies, the small $\mathrm{p}$-values for tests of heterogeneity, and the large heterogeneity $\left(I^{2}>50 \%\right)$. Limitations were due to the inclusion of original studies with risks of randomized groupings, blinding, and allocation of hidden implementation. Over the last 5 years, the downgrading of quality of evidence for outcome indicators has been significantly reduced due to the presence of limitations and inconsistencies, suggesting that researchers are gradually focusing on the randomization, blinding, and allocation concealment aspects of study design. Therefore, to address the problems of imprecision and publication bias, the author recommends that researchers should estimate the sample size, standardize the design and implementation of clinical trials, and strengthen the search for grey literature to reduce bias.
As indicated by the ROBIS tool, the risk of bias in domain 2 (identification and selection of studies) and domain 4 (synthesis and findings) was high. These results show that the range of database or electronic resources should be included in the search varying from system evaluation to systematic reviews. In addition, conference reports and clinical trial registration platforms should also be retrieved. Besides, other retrieval methods should be used, such as citation retrieval, contacting experts, tracing references and manual retrieval. Title or abstract screening must be carried out independently by at least 2 researchers. Full-texts should also be screened by at least 2 researchers to maximally minimize the bias. The funnel plot and related data analysis should be performed to determine whether there is publication bias and selective reporting.

The current review comprehensively evaluated the methodological shortcomings of the published SRs/MAs in terms of the application of acupuncture in treating KOA. As revealed by evaluation using the AMSTAR 2 tool, most of these SRs/MAs showed over 5 methodological problems, among which, 2 on average were critical items. These issues mainly contained the inclusion and exclusion criteria of studies, interpretation of bias and study pre-protocols. According to the Grading of the Quality of Evidence, limitations and publication bias were the main causes of downgrading, consistent with Li's research. ${ }^{31}$ Nevertheless, we did not find a stable improvement on the methodological quality for critical items over the years. This is mainly because that the reporting guide is primarily intended to assist authors to remember all the items that need to be reported, not to perform SRs/MAs. ${ }^{52}$ The results of this review were consistent with those from other fields (like chronic obstructive pulmonary disease (COPD), depression and hypertension). ${ }^{53-55}$ For example, Zhao et al summarized the evidence of SRs/MAs about the application of acupuncture in hypertension. They concluded that the methodological quality and quality of evidence were unsatisfactory. ${ }^{54}$ Moreover, Li et al summarized SRs/ MAs concerning the use of acupuncture in depression, and they found that the methodological quality and strength of evidence were low or critically low. ${ }^{53}$ Additionally, Chun et al assessed the methodological quality and strength of evidence of SRs/MAs regarding acupuncture in the treatment of COPD, and made the same conclusion. ${ }^{55}$ The findings suggest that there may be serious methodological problems in SRs/MAs on the use of acupuncture in the treatment of diseases. Therefore, it 
must be acknowledged that double-blinding of acupuncture trials is not feasible, and sham controls are relevant only for efficacy studies, but not for studies testing the effectiveness relative to non-treatment or to another control intervention that can be easily distinguishable from acupuncture. This problem downgrades the methodological quality and strength of evidence. Nevertheless, the quality of original studies contained is also of great importance. Hence, some items may not be well reflected and covered in the GRADE system and AMSTAR 2 tool, affecting the methodological quality and strength of evidence. Nevertheless, a number of methodological issues and the quality of outcome indicators are tightly associated with the quality of the enrolled studies, including the list and selection of inclusion/exclusion criteria, the description of baseline characteristics and conflicts of interest, the design of the study pre-protocol, and the interpretation of heterogeneity and publication bias. ${ }^{56}$ As mentioned earlier, although double-blinding of acupuncture trials is generally not feasible, investigators are required to integrate the characteristics of acupuncture diagnosis and treatment with modern clinical RCTs.

\section{Limitations}

Certain limitations should be noted in this systemic review. First, this work only summarized the findings in a narrative manner, because the interventions included in those enrolled studies varied, and the efficacy was judged upon different criteria and could not be quantitatively combined. Second, there might be bias in the assessment of AMSTAR 2, ROBIS tool and GRADE scale by different researchers, although the assessment was further evaluated and cross-checked by different researchers. Lastly, due to the small number of included studies, it was impossible to perform subgroup analyses stratified by factors like the length of treatment, different types of acupuncture, and stages of KOA.

\section{Implications for Future Practice and Research}

Our systemic review shows that acupuncture may be a promising complementary therapy beneficial for lowering pain and protecting knee joint function. To our knowledge, this review is more comprehensive, which integrates analysis based on SRs/MAs concerning the use of acupuncture in the treatment of KOA. There are several SRs/ MAs on the application of acupuncture in the treatment of
KOA. Nevertheless, upon quality of evidence and methodological quality analysis, the quality of the above contained studies was low. According to the results in this study, it is recommended that systematic evaluators should register their plans in advance at relevant websites such as PROSPERO before initiating the study, so as to avoid the potential risk of bias. Moreover, clinical researchers should improve the top-level design of clinical trials with rational evaluation and rigorous analysis. Notably, the uniform standards for clinical trial reporting (CONSORT) and the standards for reporting interventions in pinpoint clinical trials (STRICTA2010) should be employed to enhance the quality of evidence in RCTs and to improve the clinical applicability. ${ }^{57,58}$ In the future, researchers should conduct SRs with the methodological quality being controlled in line with the AMSTAR 2 checklist, ROBIS tool and GRADE evidence assessment of outcome indicators, so as to enhance the validity and scientific validity of the evidence.

\section{Conclusion}

In conclusion, the existing evidence suggests that acupuncture seems to be an effective and safe therapy for KOA. However, the significant heterogeneities in some RCTs and the deficiencies in methodological quality of the included SRs/MAs have limited the reliability of the conclusions. Therefore, further rigorous and comprehensive studies are warranted to verify the effectiveness and safety of acupuncture in KOA.

\section{Author Contributions}

All authors made a significant contribution to the work reported, whether that is in the conception, study design, execution, acquisition of data, analysis and interpretation, or in all these areas; took part in drafting, revising or critically reviewing the article; gave final approval of the version to be published; have agreed on the journal to which the article has been submitted; and agree to be accountable for all aspects of the work.

\section{Funding}

This work was supported by grants from the National Natural Science Foundation of China (NO. 81873316).

\section{Disclosure}

All authors have completed the ICMJE uniform disclosure form. The authors have no conflicts of interest to declare. 


\section{References}

1. Sharma L, Solomon CG. Osteoarthritis of the knee. $N$ Engl J Med. 2021;384(1):51-59. doi:10.1056/NEJMcp1903768

2. Sun JS, Wang WW, Lian HK. The clinical significance of changes in serum new cytokine CYTL1 in patients with knee osteoarthritis. Int J Gen Med. 2021;14:5105-5109. doi:10.2147/ijgm.S322370

3. Pigeolet M, Jayaram A, Park KB, Meara JG. Osteoarthritis in 2020 and beyond. Lancet (London, England). 2021;397 (10279):1059-1060. doi:10.1016/s0140-6736(21)00208-7

4. Bijlsma JW, Berenbaum F, Lafeber FP. Osteoarthritis: an update with relevance for clinical practice. Lancet (London, England). 2011;377 (9783):2115-2126. doi:10.1016/s0140-6736(11)60243-2

5. Kolasinski SL, Neogi T, Hochberg MC, et al. 2019 American College of Rheumatology/Arthritis Foundation guideline for the management of osteoarthritis of the hand, hip, and knee. Arthr Care Res. 2020;72 (2):149-162. doi:10.1002/acr.24131

6. da Costa BR, Reichenbach S, Keller N, et al. Effectiveness of non-steroidal anti-inflammatory drugs for the treatment of pain in knee and hip osteoarthritis: a network meta-analysis. Lancet (London, England). 2017;390(10090):e21-e33. doi:10.1016/s0140-6736(17) 31744-0

7. Solomon DH, Husni ME, Libby PA, et al. The risk of major NSAID toxicity with celecoxib, ibuprofen, or naproxen: a secondary analysis of the PRECISION trial. Am J Med. 2017;130(12):1415-1422.e1414. doi:10.1016/j.amjmed.2017.06.028

8. Chan FKL, Ching JYL, Tse YK, et al. Gastrointestinal safety of celecoxib versus naproxen in patients with cardiothrombotic diseases and arthritis after upper gastrointestinal bleeding (CONCERN): an industry-independent, double-blind, double-dummy, randomised trial. Lancet (London, England). 2017;389(10087):2375-2382. doi:10.1016/ s0140-6736(17)30981-9

9. Nissen SE, Yeomans ND, Solomon DH, et al. Cardiovascular safety of celecoxib, naproxen, or ibuprofen for arthritis. $N$ Engl $J$ Med. 2016;375(26):2519-2529. doi:10.1056/NEJMoa1611593

10. Runhaar J, Rozendaal RM, van Middelkoop M, et al. Subgroup analyses of the effectiveness of oral glucosamine for knee and hip osteoarthritis: a systematic review and individual patient data meta-analysis from the OA trial bank. Ann Rheum Dis. 2017;76 (11):1862-1869. doi:10.1136/annrheumdis-2017-211149

11. Bannuru RR, Osani MC, Vaysbrot EE, et al. OARSI guidelines for the non-surgical management of knee, hip, and polyarticular osteoarthritis. Osteoarthr Cartilage. 2019;27(11):1578-1589. doi:10.1016/j. joca.2019.06.011

12. Bruyère $\mathrm{O}$, Honvo $\mathrm{G}$, Veronese $\mathrm{N}$, et al. An updated algorithm recommendation for the management of knee osteoarthritis from the European Society for Clinical and Economic Aspects of Osteoporosis, Osteoarthritis and Musculoskeletal Diseases (ESCEO). Semin Arthritis Rheum. 2019;49 (3):337-350. doi:10.1016/j.semarthrit.2019.04.008

13. McAlindon TE, LaValley MP, Harvey WF, et al. Effect of intra-articular triamcinolone vs saline on knee cartilage volume and pain in patients with knee osteoarthritis: a randomized clinical trial. JAMA. 2017;317(19):1967-1975. doi:10.1001/jama.2017.5283

14. Rutjes AW, Jüni P, da Costa BR, Trelle S, Nüesch E, Reichenbach S. Viscosupplementation for osteoarthritis of the knee: a systematic review and meta-analysis. Ann Intern Med. 2012;157(3):180-191. doi:10.7326/0003-4819-157-3-201208070-00473

15. Gunaratne R, Pratt DN, Banda J, Fick DP, Khan RJK, Robertson BW. Patient dissatisfaction following total knee arthroplasty: a systematic review of the literature. J Arthroplasty. 2017;32(12):3854-3860. doi:10.1016/j.arth.2017.07.021

16. Arden NK, Perry TA, Bannuru RR, et al. Non-surgical management of knee osteoarthritis: comparison of ESCEO and OARSI 2019 guidelines. Nat Rev Rheumatol. 2021;17(1):59-66. doi:10.1038/ s41584-020-00523-9
17. Smith CA, Collins CT, Levett KM, et al. Acupuncture or acupressure for pain management during labour. Cochrane Database Syst Rev. 2020;2(2):Cd009232. doi:10.1002/14651858.CD009232.pub2

18. Mu J, Furlan AD, Lam WY, Hsu MY, Ning Z, Lao L. Acupuncture for chronic nonspecific low back pain. Cochrane Database Syst Rev. 2020;12(12):Cd013814. doi:10.1002/14651858.Cd013814

19. Kang HR, Lee YS, Kim SH, et al. Effectiveness and safety of electrical moxibustion for knee osteoarthritis: a multicenter, randomized, assessor-blinded, parallel-group clinical trial. Complement Ther Med. 2020;53:102523. doi:10.1016/j.ctim.2020.102523

20. Zhang Y, Wang C. Acupuncture and chronic musculoskeletal pain. Curr Rheumatol Rep. 2020;22(11):80. doi:10.1007/s11926-02000954-z

21. Venuti AJ, Chiu JP, Yu KC, Chang SC, Lin SY, Hsu CH. Chinese herbal fumigation steam therapy and acupuncture in the treatment of knee osteoarthritis: a three-armed, randomized, controlled trial. Altern Ther Health Med. 2021. doi:10.17632/t7pkdfdhg2.1

22. Tu JF, Yang JW, Shi GX, et al. Efficacy of intensive acupuncture versus sham acupuncture in knee osteoarthritis: a randomized controlled trial. Arthr Rheumatol. 2021;73(3):448-458. doi:10.1002/ art.41584

23. Wang TQ, Li YT, Wang LQ, et al. Electroacupuncture versus manual acupuncture for knee osteoarthritis: a randomized controlled pilot trial. Acupunct Med. 2020;38(5):291-300. doi:10.1177/ 0964528419900781

24. Xu S, Yu L, Luo X, et al. Manual acupuncture versus sham acupuncture and usual care for prophylaxis of episodic migraine without aura: multicentre, randomised clinical trial. BMJ (Clinical Research Ed). 2020;368:m697. doi:10.1136/bmj.m697

25. He Y, Guo X, May BH, et al. Clinical evidence for association of acupuncture and acupressure with improved cancer pain: a systematic review and meta-analysis. JAMA Oncol. 2020;6(2):271-278. doi:10.1001/jamaoncol.2019.5233

26. Zhang R, Lao L, Ren K, Berman BM. Mechanisms of acupuncture-electroacupuncture on persistent pain. Anesthesiology. 2014;120(2):482-503. doi:10.1097/aln.0000000000000101

27. Liu W, Fan Y, Wu Y, et al. Efficacy of acupuncture-related therapy in the treatment of knee osteoarthritis: a network meta-analysis of randomized controlled trials. J Pain Res. 2021;14:2209-2228. doi:10.2147/jpr.S315956

28. Sun J, Zhao Y, Zhu R, et al. Acupotomy therapy for knee osteoarthritis pain: systematic review and meta-analysis. Evid Based Compl Alter Med. 2020;2020:2168283. doi:10.1155/2020/2168283

29. Cumpston M, Li T, Page MJ, et al. Updated guidance for trusted systematic reviews: a new edition of the Cochrane Handbook for Systematic Reviews of Interventions. Cochrane Database Syst Rev. 2019;10:Ed000142. doi:10.1002/14651858.Ed000142

30. Sun N, Tu JF, Lin LL, et al. Correlation between acupuncture dose and effectiveness in the treatment of knee osteoarthritis: a systematic review. Acupunct Med. 2019;37(5):261-267. doi:10.1136/acupmed2017-011608

31. Li J, Li YX, Luo LJ, et al. The effectiveness and safety of acupuncture for knee osteoarthritis: an overview of systematic reviews. Medicine. 2019;98(28):e16301. doi:10.1097/md.0000000000016301

32. Shea BJ, Reeves BC, Wells G, et al. AMSTAR 2: a critical appraisal tool for systematic reviews that include randomised or non-randomised studies of healthcare interventions, or both. $B M J$ (Clinical Research Ed). 2017;358:j4008. doi:10.1136/bmj.j4008

33. Guyatt GH, Oxman AD, Vist GE, et al. GRADE: an emerging consensus on rating quality of evidence and strength of recommendations. BMJ (Clinical Research Ed). 2008;336 (7650):924-926. doi:10.1136/bmj.39489.470347.AD

34. Deyle GD, Allen CS, Allison SC, et al. Physical therapy versus glucocorticoid injection for osteoarthritis of the knee. $N$ Engl $J$ Med. 2020;382(15):1420-1429. doi:10.1056/NEJMoa1905877 
35. Pas HI, Winters M, Haisma HJ, Koenis MJ, Tol JL, Moen MH. Stem cell injections in knee osteoarthritis: a systematic review of the literature. Br J Sports Med. 2017;51(15):1125-1133. doi:10.1136/ bjsports-2016-096793

36. Whiting P, Savović J, Higgins JPT, et al. [ROBIS: a new tool to assess risk of bias in systematic reviews was developed.]. Recenti Prog Med. 2018;109(9):421-431. Swedish. doi:10.1701/2990.29928

37. Chai H, Lai B, Du YH. Analysis of the efficacy of acupuncture versus western medicine in the treatment of osteoarthritis of the knee. Liaoning J Trad Chin Med. 2009;36(07):1197-1200.

38. Cao L, Zhang XL, Gao YS, et al. Needle acupuncture for osteoarthritis of the knee. A systematic review and updated meta-analysis. Saudi Med J. 2012;33(5):526-532. doi:10.1001/jama.2012.4230

39. Lu M, Tan XY, Huang LU. Meta-analysis of acupuncture in the treatment of osteoarthritis of the knee joint. $J$ Trad Chin Med. 2012;18(01):81-83. doi:10.3969/j.issn.1672-951X.2012.01.037

40. Lu W. Meta-analysis of the efficacy of warm acupuncture compared with oral western medicine in the treatment of osteoarthritis of the knee. Elect J Trauma Emerg Med. 2015;3(02):30-34.

41. Lin X, Huang K, Zhu G, Huang Z, Qin A, Fan S. The effects of acupuncture on chronic knee pain due to osteoarthritis: a meta-analysis. J Bone Joint Surg Am. 2016;98(18):1578-1585. doi:10.2106/jbjs. 15.00620

42. Zhao B, Meta-analysis of the efficacy of electroacupuncture in the treatment of osteoarthritis of the knee [Thesis of master's degree]. Liaoning University of Traditional Chinese Medicine; 2016.

43. Hu HJ. Meta-analysis of acupuncture for osteoarthritis of the knee. Contemporary Nurse. 2016;9:115-117.

44. Guo KY, Chen DL. Systematic evaluation of warm acupuncture in the treatment of knee osteoarthritis. Trad Chin Med Orthopedics. 2018;30(07):17-20.

45. Cao J, Zhou Q, Qian Q. Systematic evaluation of clinical efficacy of acupuncture combined with sodium vitrate joint cavity injection versus sodium vitrate joint cavity injection alone in the treatment of knee osteoarthritis. Trad Chin Med Orthopedics. 2019;31(03):33-37.

46. Kong YY, Jing MY, Qin Y, et al. Meta-analysis of the efficacy of warm acupuncture combined with sodium vitreous acid joint cavity injection in patients with knee osteoarthritis. World Abstract Latest Med Inf. 2019;19(20):33-38+41.

47. Zheng Y, Duan X, Qi S, et al. Acupuncture therapy plus hyaluronic acid injection for knee osteoarthritis: a meta-analysis of randomized controlled trials. Evid Based Compl Alter Med. 2020;2020:4034105. doi:10.1155/2020/4034105
48. Wang TT, Liu Y, Ning ZY, Qi R. Efficacy and safety of acupuncture for the treatment of knee osteoarthritis: a systematic review and meta-analysis. $J$ Acupunct Tuina Sci. 2020;18(3):180-190. doi:10.1007/s11726-020-1177-9

49. Fan MM, Fan YM, Feng L, et al. Meta-analysis of the efficacy and safety of acupuncture in the treatment of knee osteoarthritis. Rheum Arthr. 2020;9(05):32-37+46.

50. Yang TW, Wang YL, Jiang XY. Meta-analysis of the efficacy of fire acupuncture compared with oral western medicine in the treatment of osteoarthritis of the knee. Chin Med Innovation. 2021;18(03):162-167. doi:10.3969/j.issn.1674-4985.2021.03.040

51. Yuan T, Xiong J, Wang X, et al. The quality of methodological and reporting in network meta-analysis of acupuncture and moxibustion: a cross-sectional survey. Evid Based Compl Alter Med. 2021;2021:2672173. doi:10.1155/2021/2672173

52. Moher D, Weeks L, Ocampo M, et al. Describing reporting guidelines for health research: a systematic review. J Clin Epidemiol. 2011;64(7):718-742. doi:10.1016/j.jclinepi.2010.09.013

53. Li M, Niu J, Yan P, et al. The effectiveness and safety of acupuncture for depression: an overview of meta-analyses. Complement Ther Med. 2020;50:102202. doi:10.1016/j.ctim.2019.102202

54. Zhao H, Li D, Li Y, et al. Efficacy and safety of acupuncture for hypertension: an overview of systematic reviews. Complement Ther Clin Pract. 2019;34:185-194. doi:10.1016/j.ctcp.2018.12.003

55. Chun L, Li X, Feng Z, Xie Y, Li J. Role of acupuncture in the treatment of COPD: an overview of systematic reviews. Int J Gen Med. 2021;14:1079-1092. doi:10.2147/ijgm.S300270

56. Xu C, Furuya-Kanamori L, Kwong JSW, Li S, Liu Y, Doi SA. Methodological issues of systematic reviews and meta-analyses in the field of sleep medicine: a meta-epidemiological study. Sleep Med Rev. 2021;57:101434. doi:10.1016/j.smrv.2021.101434

57. Svenkerud S, MacPherson H. The impact of STRICTA and CONSORT on reporting of randomised control trials of acupuncture: a systematic methodological evaluation. Acupunct Med. 2018;36 (6):349-357. doi:10.1136/acupmed-2017-011519

58. MacPherson H, Altman DG, Hammerschlag R, et al. Revised STandards for Reporting Interventions in Clinical Trials of Acupuncture (STRICTA): extending the CONSORT statement. Acupunct Med. 2010;28(2):83-93. doi:10.1136/aim.2009.001370
International Journal of General Medicine

\section{Publish your work in this journal}

The International Journal of General Medicine is an international, peer-reviewed open-access journal that focuses on general and internal medicine, pathogenesis, epidemiology, diagnosis, monitoring and treatment protocols. The journal is characterized by the rapid reporting of reviews, original research and clinical studies across all disease areas. The manuscript management system is completely online and includes a very quick and fair peer-review system, which is all easy to use. Visit http://www.dovepress.com/ testimonials.php to read real quotes from published authors. 